\title{
PSEUDO PAPILLARY TUMOUR OF PANCREAS
}

\author{
DR. QURATULAIN SOOMRO \\ Larkana Institute of Nuclear \\ Medicine and Radiotherapy \\ (LINAR), Larkana
}

\section{DR. KHAN MUHAMMAD}

\section{DR. ABDUL SAMAD SHAIKH}

Dr. Hafeez Soomro

Dr. Sardar Khatoon

Dr. Akhtar Ali Chandio

Article Citation:

Soomro Q, Muhammad K, Shaikh AS, Soomro H, Khatoon S, Chandio AA. Pseudo papillary tumour of pancreas. Professional Med J Sep 2009; 16(4): 609-610.

ABSTRACT: During 05 years period from January 2003 to December 2007, 45 cases of Ca. Pancreas were reported to LINAR and only one case of Pseudo Papillary Tumour of Pancreas is registered. It is a rare, low malignant potential pancreatic tumour. It has unknown pathogenesis and found in young females. We report a case of 24 years old female, who presented with pain in abdomen and low grade fever of 07 months duration. Lepratomy and excisional biopsy was done. H/P came as Pseudo Papillary Tumour of Pancreas. At presentation no any L.N. were found enlarged. Like other malignant pancreatic tumours, this neoplasm does not metastasize. Its definitive treatment is complete surgical removal, no any role of chemotherapy because it doesn't metastasized, limited role of radiotherapy in local invasive tumour or in residual mass.

\section{INTRODUCTION}

The papillary cystic and solid tumours of pancreas (PCSTP) are unusual, low malignant potential, affects young females in $2^{\text {nd }}$ or $3^{\text {rd }}$ decade of their lives, it was first introduced by Frantz in 1959, so also called Frantz Tumour.

The origin and histogenesis of this tumour is controversial. PCSTP tumour may express epithelial as well as mesenchymal markers and occasionally show exocrine and endocrine features, suggesting an origin form a stem cell. It is also suggested that the sex hormones play a role in its growth but not in its genesis.

\section{CASE}

A young patient of 24 years of age, female, married, lactating mother had complaints of abdominal pain and low grade fever for seven month. Jaundice, anaemia and lymph nodes were not revealed at the time of presentation.

\section{INVESTIGATIONS}

Following investigations performed preventively were significant

\section{- H-Pylori: +ve \\ - Ultrasound scan of abdomen}

It shows well defined, rounded area $(3.9 \times 3.3 \mathrm{~cm})$ with rounded solid components within it, seen in right lobe of liver, representing hydatid cyst of liver.

\section{C.T. SCAN ABDOMEN}

It showed well defined lesion $(2.5 \times 2.2 \mathrm{~cm})$ anterior to right kidney abutting the superior mesenteric vein on its medial aspect in close proximation to the head of pancreas and the duodenum. It showed central hypodensity with slight irregular and nodular internal margins representing of pseudo pancreatic cyst possibly arising from the uncinate process of pancreas or a duodenal duplication cyst.

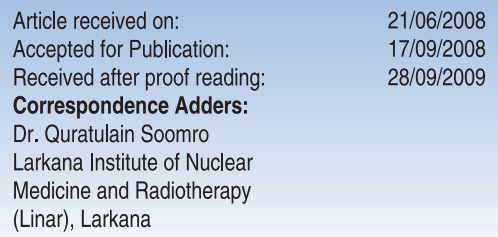




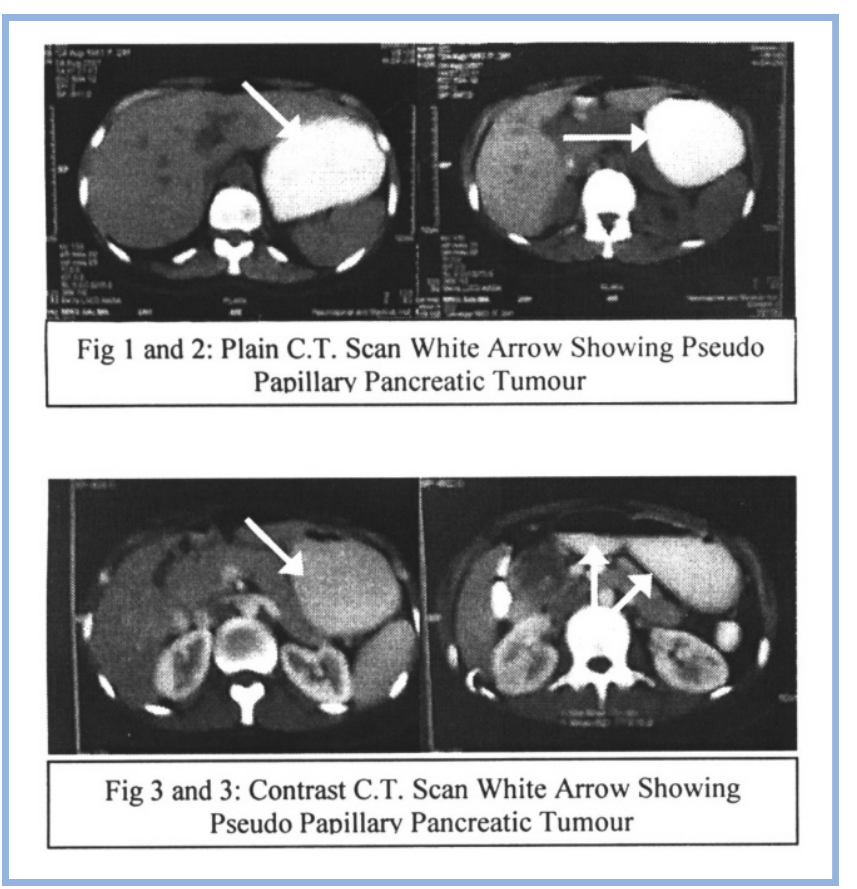

2nd opinion was taken from AKUH for C.T. Scan Which shows well defined thick walled cyst, highly suggested of hydatid cyst. However cystic neoplasia, arising from the uncinate process of pancreas could not be excluded.

\section{E. Histolytica (IHA):} 1:240 (Normal) surgery Laparotomy was done by surgeon and there was rounded, cystic cum solid vascular, swelling embedded in superficial portion of head of pancreas. Liver was found normal and lymph nodes were not seen enlarged. Excision of mass was done and sent for histopathological examination.

\section{ON GROSS EXAM}

Tan coloured, cystic tissue $(4.5 \times 4 \times 2 \mathrm{~cm})$ contains cystic solid areas.

\section{ON MICROSCOPIC EXAM}

Multiple fragments exhibiting cellular neoplastic lesion, composed of solid sheets of oval cells with ovoid nuclei with indistinct nucleoli and moderate amount of foamy cytoplasm. Focal papillary architecture is also seen. Focal cyst areas are also seen. Areas of extra cellular mucin are seen. Special stain for mucin (Alcian blue) shows positivity.
Immunohistochemical stains were also performed, which cytokeratin cam $5.2+v e$, vimentin+ve, CDIO focal +ve, synaptophysin focal +ve and Cytokeratin AE1/AE3 -ve, chromogranin -ve in tumour cells.

\section{DIAGNOSIS}

Morphological and immunohistochemical examination favours solid pseudo papillary tumour of pancreas.

\section{POST OPERATIVELY}

Patient was alright, no visceromegaly seen, Lymph node were not enlarged. Patient had tenderness at surgical scar right in the hypo chloride region.

X-ray chest, bone scan, ultrasound scan of abdomen and CEA levels were normal. She is doing well during follow up period months after surgery).

\section{CONCLUSION}

Solid Pseudo Papillary tumour of pancreas has unknown etiology with low malignant potential, occurring in young females. The sex and age suggest that hormonal factors may play role in its pathogenesis. It usually does not metastasize but patient with metastatic disease survive long. Treatment of choice is complete surgical removal with clear margins. In metastatic lesions, adjacent tissues, surgical de-bulking is necessary. Local radiotherapy to residual mass or recurrent mass. There is helpful role of chemotherapy in the primary treatment of this lesion. But in case of liver metastasis chemoemoblisation of tumour respond's well. Prognosis is excellent after complete surgical resection.

\section{ACKNOWLEDGMENTS}

We would like to express out thanks to Mr. Sajjad Ahmed Physicist/ and the technicians working at Co-60 units. Without their help this paper could be incomplete. Copyright@ 17 Sep, 2008

\section{REFERENCES}

1. Case Report at LINAR (Larkana Institute of Nuclear Medicine And Radiotherapy).

2 Abdul Kasem, Zainab Ali, Joseph Ellul. Papillary cyst and solid tumour of pancreas. Report of a case and liferature review, World Journal of Surgical Oncology, 2005; 3: 62, 1-7.

3. M.L. Gupta, Ashish H Ohri, Jasdeep Singh, Sanjeev Singla, Devinder Sing. Solid Pseudopapillary Tumour of Pancreas -A Case Report. Indian Journal of Medical \& Paediatric Oncology, 2004; Vol. 25 No. 4: 42-43. 